\title{
Iliotibial Band Syndrome
}

\section{Zeynep Demir*}

Department of Physiotherapist, Turkey

*Corresponding Author: Zeynep Demir, Department of Physiotherapist, Turkey.

Received: July 23, 2019; Published: August 16, 2019

The iliotibial band tract or IT band (ITB) is a longitudinal fibrous sheath that runs along the lateral thigh and serves as an important structure involved in lower extremity motion. The ITB is also sometimes known as Maissiat's band. The ITB spans the lower extremity on its lateral aspect before inserting on Gerdy's tubercle on the proximal/lateral tibia.

Proximally in the thigh, the ITB receives fascial contributions from the deep fascia of the thigh, gluteus maximus, and tensor fascia lata (TFL).The TFL is the deep investing fascia of the thigh, encompassing the muscles of the hip and lower extremity around this region. Distally, the ITB becomes a distinct soft tissue layer of the lateral knee.

There are multiple clinical conditions that can present secondary to a spectrum of ITB dysfunction and many of these manifest in physical laborers to recreational or high-level professional athletes. Moreover, these conditions will vary depending on the specific anatomic location of the dysfunction. For example, proximally based ITB conditions include external snapping hip syndrome, which occurs secondary to ITB friction as these fibers rub (or "snap") over the greater trochanter of the femur. Distally, ITB pathology most commonly manifests as some form of lateral-based knee pain (commonly ITB syndrome, or ITBS). Athletes affected are often involved in sports heavily reliant on peak lower extremity function and performers, such as runners, skaters, or cyclists.

Regardless of the ITB condition, most patients experience complete resolution of symptoms following nonoperative management modalities alone. However, surgical consideration is a potential option for chronic, recurrent, or recalcitrant cases that continue to persist despite exhausting of all nonoperative management options after several months.
Function

Proximal ITB function includes

- Hip extension

- Hip abduction

- Lateral hip rotation

- Distally, ITB function depends on the position of the knee joint

- 0 degrees/full extension to 20 to 30 degrees of flexion

- $\quad$ Active knee extensor

- The ITB lies anterior to the lateral femoral epicondyle

- 20 to 30 degrees of flexion to full flexion ROM

- Active knee flexor

- ITB lies posterior relative to the lateral femoral epicondyle

Knee pain has been reported to limit mobility and impair quality of life in $25 \%$ of adults. Iliotibial band syndrome (ITBS) is one of the many different causes of lateral knee pain. It was first seen in US Marine Corps recruits during their training in 1975 and has been diagnosed frequently in long distance runners, cyclists, skiers and participants of hockey, basketball, and soccer since then. These activities all depend on rapid and prolonged cycling of the knee through flexion and extension. This article will discuss how this cycling can cause the syndrome, as well as, discuss evaluation and treatment modalities.

The iliotibial band (ITB) is the distal fascial continuation of the tensor fascia lata, gluteus medius, and gluteal maximus. It traverses superficial to the vastus lateralis and inserts on the Gerdy tubercle of the lateral tibial plateau and partially to the supracondylar ridge of the lateral femur. There is also an anterior extension called the 
iliopatella band that connects the lateral patella and prevents medial translation of the patella. The ITB functions as a knee extensor when the knee is less than 30 degrees of flexion but becomes a knee flexor after exceeding 30 degrees of flexion. The ITB has been postulated to acquire a more posterior position relative to the lateral femoral epicondyle with increasing degrees of flexion.

Clinical examination testing for ITB dysfunction is best elicited utilizing the Ober Test

To perform the Ober test, have the patient lie on his or her uninvolved side in the lateral decubitus position. The symptomatic side should be facing upward (i.e., closest to the ceiling). Next, the examiner passively flexes the knee to about 90 degrees. The hip is then brought passively into a flexed and abducted position. Next, the examiner assesses the passive flexibility over the ITB with the hip brought into increasing levels of extension and adduction. A positive test entails pain, tightness, or clicking over the ITB.
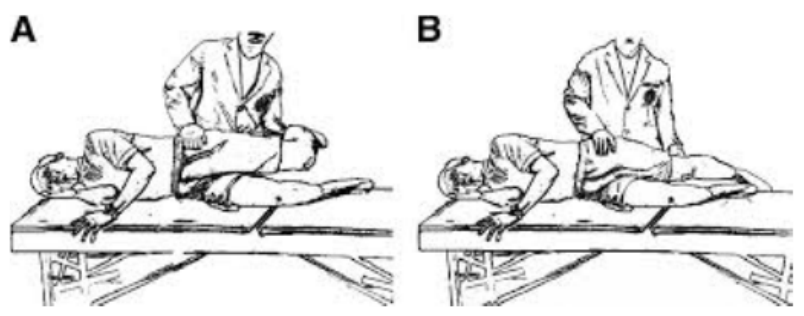

Figure 1

\section{Treatment/Management}

The first line treatment for the vast majority of patients with ITBS is nonoperative management. The patient should be advised to refrain from the inciting activity until the pain has resolved. Intermittent cryotherapy can help with acute flares. A gradual return to activity can commence as soon as the patient is pain-free with activity and palpation. Physical therapy focused on stretching of the ITB and abduction strengthening can be very effective at lengthening the ITB and reducing tension. Furthermore, manual therapy such myofascial release with a foam roller has been used to break up adhesions and is an integral part of the rehabilitation process. The physical therapist should also focus on teaching proper ergonomics and posture while performing inciting activities.

Non-steroidal anti-inflammatories can help with diminishing the inflammation. Likewise, corticosteroid injections can be therapeutic, as well as diagnostic for ITBS by providing immediate and prolonged pain relief. Shoe modification, foot orthosis, and sport specific technique training can help prevent ITBS from returning in some cases. Most patients will have complete symptom relief and able to return to activity within 6 to 8 weeks with nonoperative management alone.

The recommended progression back to activity starts with a week of running on alternating days on a flat surface. The following weeks are focused on fast-paced running daily and avoid downhill running. After 3 to 4 weeks, the patient can gradually increase the distance and frequency. Introducing hills and cambered surfaces should only take place if there is no pain or symptoms on a flat surface. If the patient relapses, then they will need to start their activity progression over again and may require a period of rest beforehand.

Surgical intervention is only for refractory cases despite nonoperative management for more than 6 months. There are several surgical options including percutaneous or open ITB release, ITB lengthening with a Z-plasty, open ITB bursectomy, and arthroscopic ITB debridement. Furthermore, there are several different techniques described for the open ITB release including resecting a triangular portion of the distal posterior ITB band, resecting the $2 \mathrm{~cm} \times 4 \mathrm{~cm}$ portion overlying the lateral femoral epicondyle and also making a V-shaped incision in the ITB.All of the different techniques have demonstrated success, but there are limited comparative studies to demonstrate superiority. ITB bursectomy is the indicated option with the symptomatology of ITBS but no enhancement of the ITB on MRI. The procedure consists of excising the underlying ITB bursa but leaving the ITB completely intact. One study demonstrated that the majority of patients who undergo open bursectomy are overall completely or mostly satisfied with the results. Minimal invasive techniques have been described to resect the lateral synovial recess that connects the ITB to the lateral femur and innervated synovial fat. 


\section{Exercise prescription}

- $\quad$ Exercises to stretch the iliotibial band and related structures.

- Myofacial treatment is ideal in acute phase, when pain and inflammation in the insertion is felt. The triggerpoints in Biceps femoris, vastus lateralis, gluteus maximus, and tensor fascia latae muscles will be addressed by a myofascial treatment.

- $\quad$ Exercises to strengthen the abductor muscles and stabilize the hip.

- Hip/knee coordination and running/cycling style modification through the increase of neuromuscular control of gait [1-4].

\section{Bibliography}

1. https://www.archives-pmr.org/article / S 0003 9993(06)00531-4/pdf

2. Andrew Hadeed and David C Tapscott. "Iliotibial Band Friction Syndrome" (2019).

3. Scott Hyland and Matthew Varacallo. "Anatomy, Bony Pelvis and Lower Limb, Iliotibial Band (Tract)" (2019).

4. https://www.physio-pedia.com/Iliotibial_Band_Syndrome

Volume 2 Issue 9 September 2019

(c) All rights are reserved by Zeynep Demir. 